CUBO A Mathematical Journal Vol.13, № 03, (141-152). October 2011

\title{
On Weak concircular Symmetries of Trans-Sasakian manifolds
}

\author{
Shyamal Kumar Hui \\ Nikhil Banga Sikshan Mahavidyalaya, \\ Bishnupur, Bankura - 722 122, \\ West Bengal, India. \\ email: shyamal_hui@yahoo.co.in
}

\begin{abstract}
The object of the present paper is to study weakly concircular symmetric and weakly concircular Ricci symmetric trans-Sasakian manifolds.
\end{abstract}

\section{RESUMEN}

El objeto del presente trabajo es el estudio de variedades simétricas débilmente concirculares y variedades simétricas trans-Sasakian débilmente concircular de Ricci.

Keywords. weakly symmetric manifold, weakly concircular symmetric manifold, weakly Ricci symmetric manifold, concircular Ricci tensor, weakly concircular Ricci symmetric manifold, $\alpha$ Sasakian manifold, $\beta$-Kenmotsu manifold, trans-Sasakian manifold.

Mathematics Subject Classification: 53C15, 53C25. 


\section{Introduction}

The notion of weakly symmetric manifolds was introduced by Tamássy and Binh [9]. A non-flat Riemannian manifold $\left(M^{n}, g\right)(n>2)$ is called a weakly symmetric manifold if its curvature tensor $R$ of type $(0,4)$ satisfies the condition

$$
\begin{aligned}
\left(\nabla_{X} R\right)(Y, Z, U, V) & =A(X) R(Y, Z, U, V)+B(Y) R(X, Z, U, V) \\
& +H(Z) R(Y, X, U, V)+D(U) R(Y, Z, X, V) \\
& +E(V) R(Y, Z, U, X)
\end{aligned}
$$

for all vector fields $X, Y, Z, U, V \in \chi\left(M^{n}\right) ; \chi(M)$ being the Lie algebra of smooth vector fields of $M$, where $A, B, H, D$ and $E$ are 1-forms (not simultaneously zero) and $\nabla$ denotes the operator of covariant differentiation with respect to the Riemannian metric g. The 1-forms are called the associated 1-forms of the manifold and an $n$-dimensional manifold of this kind is denoted by $(W S)_{n}$. In 1999 De and Bandyopadhyay [3] studied a $(W S)_{n}$ and proved that in such a manifold the associated 1-forms $B=H$ and $D=E$. Hence (1.1) reduces to the following:

$$
\begin{aligned}
\left(\nabla_{X} R\right)(Y, Z, U, V) & =A(X) R(Y, Z, U, V)+B(Y) R(X, Z, U, V) \\
& +B(Z) R(Y, X, U, V)+D(U) R(Y, Z, X, V) \\
& +\mathrm{D}(V) R(Y, Z, U, X) .
\end{aligned}
$$

A transformation of an $n$-dimensional Riemannian manifold $M$, which transforms every geodesic circle of $M$ into a geodesic circle, is called a concircular transformation [11. The interesting invariant of a concircular transformation is the concircular curvature tensor $\tilde{\mathcal{C}}$, which is defined by [1]

$$
\tilde{C}(Y, Z, U, V)=R(Y, Z, U, V)-\frac{r}{n(n-1)}[g(Z, U) g(Y, V)-g(Y, U) g(Z, V)],
$$

where $r$ is the scalar curvature of the manifold.

Recently Shaikh and Hui [7] introduced the notion of weakly concircular symmetric manifolds. A Riemannian manifold $\left(M^{n}, g\right)(n>2)$ is called weakly concircular symmetric manifold if its concircular curvature tensor $\tilde{C}$ of type $(0,4)$ is not identically zero and satisfies the condition

$$
\begin{aligned}
\left(\nabla_{X} \tilde{C}\right)(Y, Z, U, V) & =A(X) \tilde{C}(Y, Z, U, V)+B(Y) \tilde{C}(X, Z, U, V) \\
& +H(Z) \tilde{C}(Y, X, U, V)+D(U) \tilde{C}(Y, Z, X, V) \\
& +E(V) \tilde{C}(Y, Z, U, X)
\end{aligned}
$$

for all vector fields $X, Y, Z, U, V \in X\left(M^{n}\right)$, where $A, B, H, D$ and $E$ are 1-forms (not simultaneously zero) an $n$-dimensional manifold of this kind is denoted by $(W \tilde{C} S)_{n}$. Also it is shown that [7, in a $(W \tilde{C} S)_{n}$ the associated 1-forms $B=H$ and $D=E$, and hence the defining condition (1.4) of a $(W \tilde{C} S)_{n}$ reduces to the following form:

$$
\begin{aligned}
\left(\nabla_{X} \tilde{\mathrm{C}}\right)(\mathrm{Y}, \mathrm{Z}, \mathrm{U}, \mathrm{V}) & =\mathrm{A}(\mathrm{X}) \tilde{\mathrm{C}}(\mathrm{Y}, \mathrm{Z}, \mathrm{U}, \mathrm{V})+\mathrm{B}(\mathrm{Y}) \tilde{\mathrm{C}}(\mathrm{X}, \mathrm{Z}, \mathrm{U}, \mathrm{V}) \\
& +\mathrm{B}(\mathrm{Z}) \tilde{\mathrm{C}}(\mathrm{Y}, \mathrm{X}, \mathrm{U}, \mathrm{V})+\mathrm{D}(\mathrm{U}) \tilde{\mathrm{C}}(\mathrm{Y}, \mathrm{Z}, \mathrm{X}, \mathrm{V}) \\
& +\mathrm{D}(\mathrm{V}) \tilde{\mathrm{C}}(\mathrm{Y}, \mathrm{Z}, \mathrm{U}, \mathrm{X})
\end{aligned}
$$


where $A, B$ and $D$ are 1 -forms (not simultaneously zero).

Again Tamássy and Binh [10 introduced the notion of weakly Ricci symmetric manifolds. A Riemannian manifold $\left(M^{n}, g\right)(n>2)$ is called weakly Ricci symmetric manifold if its Ricci tensor $S$ of type $(0,2)$ is not identically zero and satisfies the condition

$$
\left(\nabla_{X} S\right)(Y, Z)=A(X) S(Y, Z)+B(Y) S(X, Z)+D(Z) S(Y, X)
$$

where $\mathrm{A}, \mathrm{B}$ and $\mathrm{D}$ are three non-zero 1-forms, called the associated 1-forms of the manifold, and $\nabla$ denotes the operator of covariant differentiation with respect to the metric tensor $\mathrm{g}$. Such an $\mathrm{n}$-dimensional manifold is denoted by $(\mathrm{WRS})_{\mathrm{n}}$.

Let $\left\{e_{i}: i=1,2, \cdots, n\right\}$ be an orthonormal basis of the tangent space at each point of the manifold and let

$$
\mathrm{P}(\mathrm{Y}, \mathrm{V})=\sum_{i=1}^{\mathrm{n}} \tilde{\mathrm{C}}\left(\mathrm{Y}, e_{i}, e_{i}, \mathrm{~V}\right)
$$

then from (1.3), we get

$$
P(Y, V)=S(Y, V)-\frac{r}{n} g(Y, V) .
$$

The tensor P is called the concircular Ricci symmetric tensor [4, which is a symmetric tensor of type $(0,2)$. In [4] De and Ghosh introduced the notion of weakly concircular Ricci symmetric manifolds. A Riemannian manifold $\left(M^{n}, g\right)(n>2)$ is called weakly concircular Ricci symmetric manifold [4] if its concircular Ricci tensor P of type $(0,2)$ is not identically zero and satisfies the condition

$$
\left(\nabla_{X} \mathrm{P}\right)(\mathrm{Y}, \mathrm{Z})=\mathrm{A}(\mathrm{X}) \mathrm{P}(\mathrm{Y}, \mathrm{Z})+\mathrm{B}(\mathrm{Y}) \mathrm{P}(\mathrm{X}, \mathrm{Z})+\mathrm{D}(\mathrm{Z}) \mathrm{P}(\mathrm{Y}, \mathrm{X})
$$

where $A, B$ and D are three 1-forms (not simultaneously zero).

In [5] Oubiña introduced the notion of trans-Sasakian manifolds which contains both the class of Sasakian and cosympletic structures, and are closely related to the locally conformal Kähler manifolds. A trans-Sasakian manifold of type $(0,0),(\alpha, 0)$ and $(0, \beta)$ are the cosympletic, $\alpha$-Sasakian and $\beta$-Kenmotsu manifold respectively. In particular, if $\alpha=1, \beta=0$; and $\alpha=0, \beta=1$, then a trans-Sasakian manifold reduces to a Sasakian and Kenmotsu manifold respectively. Thus transSasakian structures provide a large class of generalized quasi-Sasakian structures. Tamássy and Binh [10] studied weakly symmetric and weakly Ricci symmetric Sasakian manifolds and proved that in such a manifold the sum of the associated 1-forms vanishes everywhere. Again Özgür [6] studied weakly symmetric and weakly Ricci symmetric Kenmotsu manifolds and proved that in such a manifold the sum of the associated 1-forms is zero everywhere and hence such a manifold does not exist unless the sum of the associated 1-forms is everywhere zero.

The object of the present paper is to study weakly concircular symmetric and weakly concircular Ricci symmetric trans-Sasakian manifolds. Section 2 deals with preliminaries of trans-Sasakian manifolds. Recently Shaikh and Hui 8 studied weakly symmetric and weakly Ricci symmetric trans-Sasakian manifolds and proved that the sum of the associated 1-forms of a weakly symmetric and also of a weakly Ricci symmetric trans-Sasakian manifold of non-vanishing $\xi$-sectional curvature are non-zero everywhere and hence such two structure exists, provided that the manifold is 
of non-vanishing $\xi$-sectional curvature. However, in section 3 of the paper we have obtained all the 1-forms of a weakly concircular symmetric trans-Sasakian manifold and hence such a structure exist always. Again in section 4 we study weakly concircular Ricci symmetric trans-Sasakian manifolds and obtained all the 1-forms of a weakly concircular Ricci symmetric trans-Sasakian manifold and consequently such a structure is always exist. Also it is proved that the sum of the associated 1-forms of a weakly concircular Ricci symmetric trans-Sasakian manifold is non-vanishing everywhere.

\section{Trans-Sasakian manifolds}

A $(2 n+1)$-dimensional smooth manifold $M$ is said to be an almost contact metric manifold [1] if it admits an $(1,1)$ tensor field $\phi$, a vector field $\xi$, an 1-form $\eta$ and a Riemannian metric $\mathrm{g}$, which satisfy

$$
\begin{array}{r}
\phi \xi=0, \quad \eta(\phi X)=0, \quad \phi^{2} X=-X+\eta(X) \xi, \\
g(\phi X, Y)=-g(X, \phi Y), \quad \eta(X)=g(X, \xi), \quad \eta(\xi)=1, \\
g(\phi X, \phi Y)=g(X, Y)-\eta(X) \eta(Y)
\end{array}
$$

for all vector fields $X, Y$ on $M$.

An almost contact metric manifold $M^{2 n+1}(\phi, \xi, \eta, g)$ is said to be trans-Sasakian manifold [5] if $(M \times \mathbb{R}, J, G)$ belongs to the class $W_{4}$ of the Hermitian manifolds, where $J$ is the almost complex structure on $M \times \mathbb{R}$ defined by

$$
J\left(Z, f \frac{d}{d t}\right)=\left(\phi Z-f \xi, \eta(Z) \frac{d}{d t}\right)
$$

for any vector field $Z$ on $M$ and smooth function $f$ on $M \times \mathbb{R}$ and $G$ is the product metric on $M \times \mathbb{R}$. This may be stated by the condition $[2]$

$$
\left(\nabla_{X} \phi\right)(Y)=\alpha\{g(X, Y) \xi-\eta(Y) X\}+\beta\{g(\phi X, Y) \xi-\eta(Y) \phi X\}
$$

where $\alpha, \beta$ are smooth functions on $M$ and such a structure is said to be the trans-Sasakian structure of type $(\alpha, \beta)$. From (2.4) it follows that

$$
\begin{gathered}
\nabla_{X} \xi=-\alpha \phi X+\beta\{X-\eta(X) \xi\}, \\
\left(\nabla_{X} \eta\right)(Y)=-\alpha g(\phi X, Y)+\beta g(\phi X, \phi Y) .
\end{gathered}
$$

In a trans-Sasakian manifold $M^{2 n+1}(\phi, \xi, \eta, g)$, the following relations hold:

$$
\begin{aligned}
R(X, Y) \xi & =\left(\alpha^{2}-\beta^{2}\right)[\eta(Y) X-\eta(X) Y]-(X \alpha) \phi Y-(X \beta) \phi^{2}(Y) \\
& +2 \alpha \beta[\eta(Y) \phi X-\eta(X) \phi Y]+(Y \alpha) \phi X+(Y \beta) \phi^{2}(X)
\end{aligned}
$$




$$
\begin{aligned}
& \eta(R(X, Y) Z)=\left(\alpha^{2}-\beta^{2}\right)[g(Y, Z) \eta(X)-g(X, Z) \eta(Y)] \\
&- 2 \alpha \beta[g(\phi X, Z) \eta(Y)-g(\phi Y, Z) \eta(X)] \\
&-(Y \alpha) g(\phi X, Z)-(X \beta)\{g(Y, Z)-\eta(Y) \eta(Z)\} \\
&+(X \alpha) g(\phi Y, Z)+(Y \beta)\{g(X, Z)-\eta(Z) \eta(X)\}, \\
& S(X, \xi)=\left[2 n\left(\alpha^{2}-\beta^{2}\right)-(\xi \beta)\right] \eta(X)-((\phi X) \alpha)-(2 n-1)(X \beta), \\
& R(\xi, X) \xi=\left(\alpha^{2}-\beta^{2}-\xi \beta\right)[\eta(X) \xi-X], \\
& \quad S(\xi, \xi)=2 n\left(\alpha^{2}-\beta^{2}-\xi \beta\right), \\
&(\xi \alpha)+2 \alpha \beta=0, \\
& Q \xi=\left[2 n\left(\alpha^{2}-\beta^{2}\right)-(\xi \beta)\right] \xi+\phi(\operatorname{grad} \alpha)-(2 n-1)(\operatorname{grad} \beta),
\end{aligned}
$$

where $\mathrm{R}$ is the curvature tensor of type $(1,3)$ of the manifold and $\mathrm{Q}$ is the symmetric endomorphism of the tangent space at each point of the manifold corresponding to the Ricci tensor $S$, that is, $g(Q X, Y)=S(X, Y)$ for any vector fields $X, Y$ on $M$.

\section{Weakly concircular symmetric trans-sasakian manifolds}

Definition 3.1. A trans-Sasakian manifold $\left(M^{2 n+1}, g\right)(n>1)$ is said to be weakly concircular symmetric if its concircular curvature tensor $\tilde{\mathrm{C}}$ of type $(0,4)$ satisfies (1.5).

Setting $Y=V=e_{i}$ in (1.5) and taking summation over $i, 1 \leq i \leq 2 n+1$, we get

$$
\begin{aligned}
& \left(\nabla_{X} S\right)(Z, U)-\frac{d r(X)}{n} g(Z, U) \\
& =A(X)\left[S(Z, U)-\frac{r}{n} g(Z, U)\right]+B(Z)\left[S(X, U)-\frac{r}{n} g(X, U)\right] \\
& +D(U)\left[S(X, Z)-\frac{r}{n} g(X, Z)\right]+B(R(X, Z) U)+D(R(X, U) Z) \\
& -\frac{r}{n(n-1)}[\{B(X)+D(X)\} g(Z, U)-B(Z) g(X, U)-D(U) g(Z, X)] .
\end{aligned}
$$

Plugging $X=Z=U=\xi$ in (3.1) and then using (2.7) and (2.11), we obtain

$$
A(\xi)+B(\xi)+D(\xi)=\frac{2 n^{2}\{2 \alpha(\xi \alpha)-2 \beta(\xi \beta)-(\xi(\xi \beta))\}-d r(\xi)}{2 n^{2}\left\{\alpha^{2}-(\xi \beta)-\beta^{2}\right\}-r} .
$$

This leads to the following:

Theorem 3.1. In a weakly concircular symmetric trans-Sasakian manifold $\left(M^{2 n+1}, g\right)(n>1)$, the relation (3.2) holds. 
Next, substituting $X$ and $Z$ by $\xi$ in (3.1) and then using (2.7) and (2.12) we obtain

$$
\begin{aligned}
& \left(\nabla_{\xi} S\right)(\xi, U)-\frac{d r(\xi)}{n} \eta(U) \\
& =[A(\xi)+B(\xi)]\left[S(U, \xi)-\frac{r}{n} \eta(U)\right]+D(U)\left[( 2 n - 1 ) \left\{\alpha^{2}-(\xi \beta)\right.\right. \\
& \left.\left.-\beta^{2}\right\}-\frac{n-2}{n(n-1)} r\right]+\left[\alpha^{2}-(\xi \beta)-\beta^{2}-\frac{r}{n(n-1)}\right] \eta(U) D(\xi) .
\end{aligned}
$$

From (2.9), we have

$$
\begin{aligned}
\left(\nabla_{\xi} \mathrm{S}\right)(\xi, \mathrm{U})= & \nabla_{\xi} \mathrm{S}(\xi, \mathrm{U})-\mathrm{S}\left(\nabla_{\xi} \xi, \mathrm{U}\right)-\mathrm{S}\left(\xi, \nabla_{\xi} \mathrm{U}\right) \\
= & \nabla_{\xi} \mathrm{S}(\xi, \mathrm{U})-\mathrm{S}\left(\xi, \nabla_{\xi} \mathrm{U}\right) \\
= & {[2 \mathrm{n}\{2 \alpha(\xi \alpha)-2 \beta(\xi \beta)\}-(\xi(\xi \beta))] \eta(\mathrm{U}) } \\
& -(2 \mathrm{n}-1)(\mathrm{U}(\xi \beta))-(\phi \mathrm{U}(\xi \alpha)) .
\end{aligned}
$$

By virtue of (3.3) and (3.4) we obtain from (3.2) that

$$
\begin{aligned}
\mathrm{D}(\mathrm{U})= & \frac{\left[2 \mathrm{n}\{2 \alpha(\xi \alpha)-2 \beta(\xi \beta)\}-(\xi(\xi \beta))-\frac{\mathrm{dr}(\xi)}{\mathrm{n}}\right] \eta(\mathrm{U})}{(2 n-1)\left[\alpha^{2}-(\xi \beta)-\beta^{2}\right]-\frac{n-2}{n(n-1)} r} \\
- & \frac{(2 n-1)(\mathrm{U}(\xi \beta))+(\phi \mathrm{U}(\xi \alpha))}{(2 n-1)\left[\alpha^{2}-(\xi \beta)-\beta^{2}\right]-\frac{n-2}{n(n-1)} r} \\
+ & \mathrm{D}(\xi)\left[\frac{(2 n-1)\left\{\left(\alpha^{2}-\beta^{2}\right) \eta(\mathrm{U})-(\mathrm{U} \beta)\right\}-((\phi \mathrm{U}) \alpha)-\frac{n-2}{n(n-1)} r \eta(\mathrm{U})}{(2 n-1)\left\{\alpha^{2}-(\xi \beta)-\beta^{2}\right\}-\frac{n-2}{n(n-1)} r}\right] \\
- & \frac{2 n\{2 \alpha(\xi \alpha)-2 \beta(\xi \beta)-(\xi(\xi \beta))\}-\frac{\mathrm{dr}(\xi)}{n}}{\left[2 n\left\{\alpha^{2}-(\xi \beta)-\beta^{2}\right\}-\frac{r}{n}\right]\left[(2 n-1)\left\{\alpha^{2}-(\xi \beta)-\beta^{2}\right\}-\frac{n-2}{n(n-1)} r\right]} \\
& {\left[\left\{2 n\left(\alpha^{2}-\beta^{2}\right)-(\xi \beta)-\frac{r}{n}\right\} \eta(U)-(2 n-1)(\mathrm{U} \beta)-((\phi U) \alpha)\right] . }
\end{aligned}
$$

Next, setting $X=U=\xi$ in (3.1) and proceeding in a similar manner as above, we get

$$
\begin{aligned}
B(Z)= & \frac{\left[2 n\{2 \alpha(\xi \alpha)-2 \beta(\xi \beta)\}-(\xi(\xi \beta))-\frac{\mathrm{dr}(\xi)}{n}\right] \eta(Z)}{(2 n-1)\left[\alpha^{2}-(\xi \beta)-\beta^{2}\right]-\frac{n-2}{n(n-1)} r} \\
- & \frac{(2 n-1)(Z(\xi \beta))+(\phi Z(\xi \alpha))}{(2 n-1)\left[\alpha^{2}-(\xi \beta)-\beta^{2}\right]-\frac{n-2}{n(n-1)} r} \\
+ & B(\xi)\left[\frac{(2 n-1)\left\{\left(\alpha^{2}-\beta^{2}\right) \eta(Z)-(Z \beta)\right\}-((\phi Z) \alpha)-\frac{n-2}{n(n-1)} \eta \eta(Z)}{(2 n-1)\left\{\alpha^{2}-(\xi \beta)-\beta^{2}\right\}-\frac{n-2}{n(n-1)} r}\right] \\
- & \frac{2 n\{2 \alpha(\xi \alpha)-2 \beta(\xi \beta)-(\xi(\xi \beta))\}-\frac{d r(\xi)}{n}}{\left[2 n\left\{\alpha^{2}-(\xi \beta)-\beta^{2}\right\}-\frac{r}{n}\right]\left[(2 n-1)\left\{\alpha^{2}-(\xi \beta)-\beta^{2}\right\}-\frac{n-2}{n(n-1)} r\right]} \\
& {\left[\left\{2 n\left(\alpha^{2}-\beta^{2}\right)-(\xi \beta)-\frac{r}{n}\right\} \eta(Z)-(2 n-1)(Z \beta)-((\phi Z) \alpha)\right] . }
\end{aligned}
$$


Again, setting $Z=U=\xi$ in (3.1), we get

$$
\begin{aligned}
(\nabla \times S)(\xi, \xi)-\frac{\mathrm{dr}(X)}{n} & =A(X)\left[S(\xi, \xi)-\frac{r}{n}\right]+[B(\xi)+D(\xi)][S(X, \xi) \\
& \left.-\frac{n-2}{n(n-1)} r(X)\right]+B(R(X, \xi) \xi) \\
& +D(R(X, \xi) \xi)-\frac{r}{n(n-1)}[B(X)+D(X)] \\
& =\left[2 n\left\{\alpha^{2}-(\xi \beta)-\beta^{2}\right\}-\frac{r}{n}\right] A(X)+[B(\xi) \\
& +D(\xi)]\left[S(X, \xi)-\left\{\frac{n-2}{n(n-1)} r+\alpha^{2}-(\xi \beta)-\beta^{2}\right\} \eta(X)\right] \\
& +[B(X)+D(X)]\left[\alpha^{2}-(\xi \beta)-\beta^{2}-\frac{r}{n(n-1)}\right] .
\end{aligned}
$$

Now we have

$$
\left(\nabla_{X} S\right)(\xi, \xi)=\nabla_{X} S(\xi, \xi)-2 S\left(\nabla_{X} \xi, \xi\right),
$$

which yields by using (2.5) and (2.9) that

$$
\begin{aligned}
\left(\nabla_{X} S\right)(\xi, \xi) & =2 \mathfrak{n}[2 \alpha(X \alpha)-2 \beta(X \beta)-(X(\xi \beta))] \\
& +2 \alpha[(X \alpha)-\eta(X)(\xi \alpha)-(2 \mathfrak{n}-1)((\phi X) \beta)] \\
& +2 \beta[((\phi X) \alpha)+(2 \mathfrak{n}-1)\{(X \beta)-(\xi \beta) \eta(X)\}] .
\end{aligned}
$$

In view of (3.8), (3.7) yields

$$
\begin{aligned}
& {\left[2 n\left\{\alpha^{2}-(\xi \beta)-\beta^{2}\right\}-\frac{r}{n}\right] A(X)+\left[\alpha^{2}-(\xi \beta)-\beta^{2}-\frac{r}{n(n-1)}\right][B(X)+D(X)]} \\
& =2 n[2 \alpha(X \alpha)-2 \beta(X \beta)-(X(\xi \beta))]+2 \alpha[(X \alpha)-\eta(X)(\xi \alpha) \\
& -(2 n-1)((\phi X) \beta)]+2 \beta[((\phi X) \alpha)+(2 n-1)\{(X \beta)-(\xi \beta) \eta(X)\}] \\
& -\frac{d r(X)}{n}-\{B(\xi)+D(\xi)\}\left[\left\{(2 n-1)\left(\alpha^{2}-\beta^{2}\right)\right.\right. \\
& \left.\left.-\frac{n-2}{n(n-1)} r\right\} \eta(X)-((\phi X) \alpha)-(2 n-1)(X \beta)\right] .
\end{aligned}
$$


Using (3.5) and (3.6) in (3.9), we obtain

$$
\begin{aligned}
& {\left[2 n\left\{\alpha^{2}-(\xi \beta)-\beta^{2}\right\}-\frac{r}{n}\right] A(X)} \\
& =2 n[2 \alpha(X \alpha)-2 \beta(X \beta)-(X(\xi \beta))]+2 \alpha[(X \alpha)-\eta(X)(\xi \alpha) \\
& -(2 n-1)((\phi X) \beta)]+2 \beta[((\phi X) \alpha)+(2 n-1)\{(X \beta)-(\xi \beta) \eta(X)\}]-\frac{\operatorname{dr}(X)}{n} \\
& -\frac{2 n\{2 \alpha(\xi \alpha)-2 \beta(\xi \beta)-(\xi(\xi \beta))\}-\frac{\mathrm{dr}(\xi)}{n}}{(2 n-1)\left\{\alpha^{2}-(\xi \beta)-\beta^{2}\right\}-\frac{n-2}{n(n-1)} r}\left[\left\{( 2 n - 1 ) \left(\alpha^{2}\right.\right.\right. \\
& \left.\left.\left.-\beta^{2}\right)-\frac{n-2}{n(n-1)} r\right\} \eta(X)-((\phi X) \alpha)-(2 n-1)(X \beta)\right] \\
& +A(\xi) \frac{2 n\left\{\alpha^{2}-(\xi \beta)-\beta^{2}\right\}-\frac{r}{n}}{(2 n-1)\left\{\alpha^{2}-(\xi \beta)-\beta^{2}\right\}-\frac{n-2}{n(n-1)} r}\left[\left\{( 2 n - 1 ) \left(\alpha^{2}\right.\right.\right. \\
& \left.\left.\left.-\beta^{2}\right)-\frac{n-2}{n(n-1)} r\right\} \eta(X)-((\phi X) \alpha)-(2 n-1)(X \beta)\right] \\
& -\frac{2\left\{\alpha^{2}-(\xi \beta)-\beta^{2}-\frac{r}{n(n-1)}\right\}}{(2 n-1)\left\{\alpha^{2}-(\xi \beta)-\beta^{2}\right\}-\frac{n-2}{n(n-1)} r}[2 n\{2 \alpha(\xi \alpha) \\
& \left.-2 \beta(\xi \beta)\}-(\xi(\xi \beta))-\frac{\mathrm{dr}(\xi)}{\mathrm{n}}\right] \eta(X) \\
& +\frac{2\left\{\alpha^{2}-(\xi \beta)-\beta^{2}-\frac{r}{n(n-1)}\right\}}{(2 n-1)\left\{\alpha^{2}-(\xi \beta)-\beta^{2}\right\}-\frac{n-2}{n(n-1)} r}[(2 n-1)(X(\xi \beta))+(\phi X(\xi \alpha))] \\
& +\frac{2\left\{\alpha^{2}-(\xi \beta)-\beta^{2}-\frac{r}{n(n-1)}\right\}\left[2 n\{2 \alpha(\xi \alpha)-2 \beta(\xi \beta)-(\xi(\xi \beta))\}-\frac{d r(\xi)}{n}\right]}{\left[2 n\left\{\alpha^{2}-(\xi \beta)-\beta^{2}\right\}-\frac{r}{n}\right]\left[(2 n-1)\left\{\alpha^{2}-(\xi \beta)-\beta^{2}\right\}-\frac{n-2}{n(n-1)} r\right]} \\
& {\left[\left\{2 n\left(\alpha^{2}-\beta^{2}\right)-(\xi \beta)-\frac{r}{n}\right\} \eta(X)-(2 n-1)(X \beta)-((\phi X) \alpha)\right] \text {. }}
\end{aligned}
$$

This leads to the following:

Theorem 3.2. In a weakly concircular symmetric trans-Sasakian manifold $\left(M^{2 n+1}, g\right)(n>1)$, the associated 1-forms D, B and A are given by (3.5), (3.6) and (3.10) respectively.

\section{Weakly concircular Ricci symmetric trans-Sasakian man- ifolds}

Definition 4.1. A trans-Sasakian manifold $\left(M^{2 n+1}, \mathrm{~g}\right)(\mathrm{n}>1)$ is said to be weakly concircular Ricci symmetric if its concircular Ricci tensor $\mathrm{P}$ of type $(0,2)$ satisfies (1.9)). 
In view of (1.8), (1.9) yields

$$
\begin{aligned}
\left(\nabla_{X} S\right)(Y, Z)-\frac{\mathrm{dr}(X)}{n} g(Y, Z) & =A(X)\left[S(Y, Z)-\frac{r}{n} g(Y, Z)\right] \\
& +B(Y)\left[S(X, Z)-\frac{r}{n} g(X, Z)\right] \\
& +D(Z)\left[S(X, Y)-\frac{r}{n} g(X, Y)\right]
\end{aligned}
$$

Setting $X=Y=Z=\xi$ in (4.1), we get the relation (3.2) and hence we can state the following:

Theorem 4.1. In a weakly concircular Ricci symmetric trans-Sasakian manifold $\left(M^{2 n+1}, g\right)(n>$ 1), the relation (3.2) holds.

Next, substituting $X$ and $Y$ by $\xi$ in (4.1), we obtain

$$
\begin{aligned}
\left(\nabla_{\xi} S\right)(\xi, Z)-\frac{\mathrm{dr}(\xi)}{n} \eta(Z) & =[A(\xi)+B(\xi)][S(\xi, Z) \\
& \left.-\frac{r}{n} \eta(Z)\right]+D(Z)\left[S(\xi, \xi)-\frac{r}{n}\right]
\end{aligned}
$$

Using (3.2) and (3.4) in (4.2), we get

$$
\begin{aligned}
D(Z) & =\frac{\left[2 n\{2 \alpha(\xi \alpha)-2 \beta(\xi \beta)\}-(\xi(\xi \beta))-\frac{\mathrm{d} r(\xi)}{n}\right] \eta(Z)}{2 n\left[\alpha^{2}-(\xi \beta)-\beta^{2}\right]-\frac{r}{n}} \\
& -\frac{(2 n-1)(Z(\xi \beta))+(\phi Z(\xi \alpha))}{2 n\left[\alpha^{2}-(\xi \beta)-\beta^{2}\right]-\frac{r}{n}} \\
& +D(\xi)\left[\frac{2 n\left\{\left(\alpha^{2}-\beta^{2}\right)-(\xi \beta)-\frac{r}{n}\right\} \eta(Z)-((\phi Z) \alpha)-(2 n-1)(Z \beta)}{2 n\left\{\alpha^{2}-(\xi \beta)-\beta^{2}\right\}-\frac{r}{n}}\right] \\
& -\frac{2 n\{2 \alpha(\xi \alpha)-2 \beta(\xi \beta)-(\xi(\xi \beta))\}-\frac{d r(\xi)}{n}}{\left[2 n\left\{\alpha^{2}-(\xi \beta)-\beta^{2}\right\}-\frac{r}{n}\right]^{2}}\left[\left\{2 n\left(\alpha^{2}-\beta^{2}\right)\right.\right. \\
& \left.\left.-(\xi \beta)-\frac{r}{n}\right\} \eta(Z)-(2 n-1)(Z \beta)-((\phi Z) \alpha)\right] \quad \text { for all Z. }
\end{aligned}
$$

Again putting $X=Z=\xi$ in (4.1) and proceeding in a similar manner as above we get

$$
\begin{aligned}
B(Y) & =\frac{\left[2 n\{2 \alpha(\xi \alpha)-2 \beta(\xi \beta)\}-(\xi(\xi \beta))-\frac{\operatorname{dr}(\xi)}{n}\right] \eta(Y)}{2 n\left[\alpha^{2}-(\xi \beta)-\beta^{2}\right]-\frac{r}{n}} \\
& -\frac{(2 n-1)(Y(\xi \beta))+(\phi Y(\xi \alpha))}{2 n\left[\alpha^{2}-(\xi \beta)-\beta^{2}\right]-\frac{r}{n}} \\
& +B(\xi)\left[\frac{2 n\left\{\left(\alpha^{2}-\beta^{2}\right)-(\xi \beta)-\frac{r}{n}\right\} \eta(Y)-((\phi Y) \alpha)-(2 n-1)(Y \beta)}{2 n\left\{\alpha^{2}-(\xi \beta)-\beta^{2}\right\}-\frac{r}{n}}\right] \\
& -\frac{2 n\{2 \alpha(\xi \alpha)-2 \beta(\xi \beta)-(\xi(\xi \beta))\}-\frac{\operatorname{dr}(\xi)}{n}}{\left[2 n\left\{\alpha^{2}-(\xi \beta)-\beta^{2}\right\}-\frac{r}{n}\right]^{2}}\left[\left\{2 n\left(\alpha^{2}-\beta^{2}\right)\right.\right. \\
& \left.\left.-(\xi \beta)-\frac{r}{n}\right\} \eta(Y)-(2 n-1)(Y \beta)-((\phi Y) \alpha)\right] \quad \text { for all } Y .
\end{aligned}
$$


Again, setting $Y=Z=\xi$ in (4.1) and using (2.9) and (2.11), we get

$$
\begin{aligned}
\left(\nabla_{X} S\right)(\xi, \xi)-\frac{\mathrm{dr}(X)}{n} & =\left[2 n\left\{\alpha^{2}-(\xi \beta)-\beta^{2}\right\}-\frac{r}{n}\right] A(X) \\
& +[B(\xi)+D(\xi)]\left[\left\{2 n\left(\alpha^{2}-\beta^{2}\right)-(\xi \beta)\right\} \eta(X)\right. \\
& -((\phi X) \alpha)-(2 n-1)(X \beta)] .
\end{aligned}
$$

Using (3.2) and (3.8) in (4.5), we get

$$
\begin{aligned}
A(X) & =\frac{2 n[2 \alpha(X \alpha)-2 \beta(X \beta)-(X(\xi \beta))]}{2 n\left\{\alpha^{2}-(\xi \beta)-\beta^{2}\right\}-\frac{r}{n}} \\
& +\frac{2 \alpha[(X \alpha)-\eta(X)(\xi \alpha)-(2 n-1)((\phi X) \beta)]}{2 n\left\{\alpha^{2}-(\xi \beta)-\beta^{2}\right\}-\frac{r}{n}} \\
& +\frac{2 \beta[((\phi X) \alpha)+(2 n-1)\{(X \beta)-(\xi \beta) \eta(X)\}]}{2 n\left\{\alpha^{2}-(\xi \beta)-\beta^{2}\right\}-\frac{r}{n}} \\
& +A(\xi)\left[\frac{\left[2 n\left(\alpha^{2}-\beta^{2}\right)-(\xi \beta)-\frac{r}{n}\right\} \eta(X)-((\phi X) \alpha)-(2 n-1)(X \beta)}{2 n\left\{\alpha^{2}-(\xi \beta)-\beta^{2}\right\}-\frac{r}{n}}\right] \\
& -\frac{2 n\{2 \alpha(\xi \alpha)-2 \beta(\xi \beta)-(\xi(\xi \beta))\}-\frac{\operatorname{dr}(\xi)}{n}}{\left[2 n\left\{\alpha^{2}-(\xi \beta)-\beta^{2}\right\}-\frac{r}{n}\right]^{2}}\left[\left\{2 n\left(\alpha^{2}-\beta^{2}\right)\right.\right. \\
& \left.\left.-(\xi \beta)-\frac{r}{n}\right\} \eta(X)-(2 n-1)(X \beta)-((\phi X) \alpha)\right] \quad \text { for all } X .
\end{aligned}
$$

This leads to the following:

Theorem 4.2. In a weakly concircular Ricci symmetric trans-Sasakian manifold $\left(M^{2 n+1}, g\right)(n>$ 1), the associated 1-forms $\mathrm{D}, \mathrm{B}$ and $\mathrm{A}$ are given by (4.3), (4.4) and (4.6) respectively.

Adding (4.3), (4.4) and (4.6) and using (3.2), we get

$$
\begin{aligned}
& A(X)+B(X)+D(X) \\
& =\frac{2 n[2 \alpha(X \alpha)-2 \beta(X \beta)-(X(\xi \beta))]}{2 n\left\{\alpha^{2}-(\xi \beta)-\beta^{2}\right\}-\frac{r}{n}} \\
& +\frac{2 \alpha[(X \alpha)-\eta(X)(\xi \alpha)-(2 n-1)((\phi X) \beta)]}{2 n\left\{\alpha^{2}-(\xi \beta)-\beta^{2}\right\}-\frac{r}{n}} \\
& +\frac{2 \beta[((\phi X) \alpha)+(2 n-1)\{(X \beta)-(\xi \beta) \eta(X)\}]}{2 n\left\{\alpha^{2}-(\xi \beta)-\beta^{2}\right\}-\frac{r}{n}} \\
& \frac{2\left[2 n\{2 \alpha(\xi \alpha)-2 \beta(\xi \beta)\}-(\xi(\xi \beta))-\frac{d r(\xi)}{n}\right] \eta(X)}{2 n\left\{\alpha^{2}-(\xi \beta)-\beta^{2}\right\}-\frac{r}{n}} \\
& -\frac{(2 n-1)(X(\xi \beta))+(\phi X(\xi \alpha))}{n\left\{\alpha^{2}-(\xi \beta)-\beta^{2}\right\}-\frac{r}{2 n}} \\
& -\frac{2\left[2 n\{2 \alpha(\xi \alpha)-2 \beta(\xi \beta)-(\xi(\xi \beta))\}-\frac{d r(\xi)}{n}\right]}{\left[2 n\left\{\alpha^{2}-(\xi \beta)-\beta^{2}\right\}-\frac{r}{n}\right]^{2}}\left[\left\{2 n\left(\alpha^{2}-\beta^{2}\right)\right.\right. \\
& \left.\left.-(\xi \beta)-\frac{r}{n}\right\} \eta(X)-(2 n-1)(X \beta)-((\phi X) \alpha)\right]
\end{aligned}
$$


for any vector field $X$. This leads to the following:

Theorem 4.3. In a weakly concircular Ricci symmetric trans-Sasakian manifold $\left(M^{2 n+1}, \mathrm{~g}\right)(\mathrm{n}>$ 1 ), the sum of the associated 1-forms is given by (4.7).

In particular, if $\phi(\operatorname{grad} \alpha)=\operatorname{grad} \beta$, then $(\xi \beta)=0$ and hence the relation (4.7) reduces to the following form

$$
\begin{aligned}
& A(X)+B(X)+D(X) \\
& =\frac{2 n[2 \alpha(X \alpha)-2 \beta(X \beta)]}{2 n\left(\alpha^{2}-\beta^{2}\right)-\frac{r}{n}}+\frac{2 \alpha\{(X \alpha)-\eta(X)(\xi \alpha)-(2 n-1)((\phi X) \beta)\}}{2 n\left(\alpha^{2}-\beta^{2}\right)-\frac{r}{n}} \\
& +\frac{2 \beta\{((\phi X) \alpha)+(2 n-1)(X \beta)\}+2\left\{4 n \alpha(\xi \alpha)-\frac{d r(\xi)}{n}\right\} \eta(X)-2(\phi X(\xi \alpha))}{2 n\left(\alpha^{2}-\beta^{2}\right)-\frac{r}{n}} \\
& -\frac{2\left[4 n \alpha(\xi \alpha)-\frac{d r(\xi)}{n}\right]}{\left[2 n\left(\alpha^{2}-\beta^{2}\right)-\frac{r}{n}\right]^{2}}\left[\left\{2 n\left(\alpha^{2}-\beta^{2}\right)-\frac{r}{n}\right\} \eta(X)-((\phi X) \alpha)-(2 n-1)(X \beta)\right] .
\end{aligned}
$$

for any vector field $X$. This leads to the following:

Corollary 4.1. If a weakly concircular Ricci symmetric trans-Sasakian manifold $\left(M^{2 n+1}, \mathrm{~g}\right)(\mathrm{n}>$ 1) satisfies the condition $\phi(\operatorname{grad} \alpha)=\operatorname{grad} \beta$, then the sum of the associated 1 -forms is given by (4.8).

If $\beta=0$ and $\alpha=1$, then (4.7) yields $A(X)+B(X)+D(X)=0$ for all $X$ and hence we can state the following:

Corollary 4.2. There is no weakly concircular Ricci symmetric Sasakian manifold $M^{2 n+1}(n>1)$, unless the sum of the 1-forms is everywhere zero.

Corollary 4.3. If an $\alpha$-Sasakian manifold is weakly concircular Ricci symmetric, then the sum of the 1-forms, i.e., $\mathrm{A}+\mathrm{B}+\mathrm{D}$ is given by

$$
\begin{aligned}
A(X)+B(X)+D(X) & =\frac{2 \alpha[(2 n+1)(X \alpha)-\eta(X)(\xi \alpha)]-2(\phi X(\xi \alpha))}{2 n \alpha^{2}-\frac{r}{n}} \\
& +\frac{2\left[4 n \alpha(\xi \alpha)-\frac{\mathrm{d} r(\xi)}{n}\right]((\phi X) \alpha)}{\left(2 n \alpha^{2}-\frac{r}{n}\right)^{2}}
\end{aligned}
$$

Again, if $\alpha=0$ and $\beta=1$, then (4.7) yields $A(X)+B(X)+D(X)=0$ for all $X$. This leads to the following:

Corollary 4.4. There is no weakly concircular Ricci symmetric Kenmotsu manifold $M^{2 n+1}(n>$ 1), unless the sum of the 1-forms is everywhere zero.

Corollary 4.5. If a $\beta$-Kenmotsu manifold is weakly concircular Ricci symmetric, then the sum of 
the 1-forms, i.e., $\mathrm{A}+\mathrm{B}+\mathrm{D}$ is given by

$$
\begin{aligned}
& A(X)+B(X)+D(X) \\
& =\frac{2 n\{2 \beta(X \beta)+(X(\xi \beta))\}-2(2 n-1) \beta\{(X \beta)-(\xi \beta) \eta(X)\}}{2 n\left\{(\xi \beta)+\beta^{2}\right\}+\frac{r}{n}} \\
& +\frac{2\left[\left\{4 n \beta(\xi \beta)+\left(\xi(\xi \beta)+\frac{d r(\xi)}{n}\right\} \eta(X)+(2 n-1)(X(\xi \beta))\right]\right.}{2 n\left\{(\xi \beta)+\beta^{2}\right\}+\frac{r}{n}} \\
& -\frac{2\left[2 n\{2 \beta(\xi \beta)+(\xi(\xi \beta))\}+\frac{d r(\xi)}{n}\right]\left[\left\{2 n \beta^{2}+(\xi \beta)+\frac{r}{n}\right\} \eta(X)+(2 n-1)(X \beta)\right]}{\left[2 n\left\{(\xi \beta)+\beta^{2}\right\}+\frac{r}{n}\right]^{2}} . \\
& \text { Received: April 2010. Revised: September 2010. }
\end{aligned}
$$

\section{References}

[1] Blair, D. E., Contact manifolds in Riemannian geometry, Lecture Notes in Math. 509, Springer-Verlag, 1976.

[2] Blair, D. E. and Oubina, J. A., Conformal and related changes of metric on the product of two almost contact metric manifolds, Publ. Math. Debrecen, 34 (1990), 199-207.

[3] De, U. C. and Bandyopadhyay, S., On weakly symmetric Riemannian spaces, Publ. Math. Debrecen, 54/3-4 (1999), 377-381.

[4] De, U. C. and Ghosh, G. C., On weakly concircular Ricci symmetric manifolds, South East Asian J. Math. and Math. Sci., 3(2) (2005), 9-15.

[5] Oubina, J. A., New class of almost contact metric manifolds, Publ. Math. Debrecen, 32 (1985), 187-193.

[6] Özgür, C., On weakly symmetric Kenmotsu manifolds, Diff. Geom.-Dynamical Systems, 8 (2006), 204-209.

[7] Shaikh, A. A. and Hui, S. K., On weakly concircular symmetric manifolds, Ann. Sti. Ale Univ., "Al. I. CUZA", Din Iasi, LV, f.1 (2009), 167-186.

[8] Shaikh, A. A. and Hui, S. K., On weak symmetries of trans-Sasakian manifolds, Proc. Estonian Acad. Sci., 58(4) (2009), 213-223.

[9] Tamássy, L. and Binh, T. Q., On weakly symmetric and weakly projective symmetric Rimannian manifolds, Coll. Math. Soc., J. Bolyai, 56 (1989), 663-670.

[10] Tamássy, L. and Binh, T. Q., On weak symmetries of Einstein and Sasakian manifolds, Tensor N. S., 53 (1993), 140-148.

[11] Yano, K., Concircular geometry I, concircular transformations, Proc. Imp. Acad. Tokyo, 16 (1940), 195-200. 